\title{
Perceptions, beliefs and societal factors impacting on exclusive breastfeeding of primiparous mothers: An exploratory study
}

\author{
Fahima Al Harthy*1, Asma Al Zaabi ${ }^{1}$, Najat Al Ajmi ${ }^{1}$, Afaf Elshiekh ${ }^{1}$, Kamila Al Alawi ${ }^{2}$, Lamia Mahmoud ${ }^{2}$, Mudhar \\ Al Adawi ${ }^{1}$ \\ ${ }^{1}$ Royal Hospital, Ministry of Health (MOH), Oman \\ ${ }^{2}$ Technical Officer, WHO-Oman, Oman
}

Received: September 23, 2021

DOI: $10.5430 / \mathrm{cns} . v 9 \mathrm{n} 2 \mathrm{p} 30$
Accepted: October 26, 2021

Online Published: November 1, 2021

\begin{abstract}
This study aims to explore the perceptions of Omani primiparous mothers towards exclusive breastfeeding. A qualitative exploratory study was conducted at Royal Hospital between March and November 2020. Purposive sampling was applied to recruit newly delivered mothers and face to face interview was done for data collection. Interview protocol along with prompts was used to guide researchers during the interview. Specific questions were administered to assess mothers' knowledge of exclusive breastfeeding, sources of information, influencing factors toward exclusive breastfeeding and intention to exclusively breastfeeding. Thematic analysis revealed six themes, namely: the effects of lack of knowledge on the mother perception towards exclusive $\mathrm{BF}$, family members influencing the decision-making process regarding $\mathrm{BF}$, impact of social life on $\mathrm{BF}$ practices, the effect of mother's beliefs on exclusive BF, factor that affect BF practice in early days, mother's concern regarding emotional support for successful exclusive BF. This study revealed a lack of basic knowledge on exclusive breastfeeding among mothers. Moreover, grandmothers and husbands were found to be the most influential family members on the decision regarding exclusive breastfeeding. Mothers had major concerns regarding milk adequacy in the first few days after delivery and emotional support plays a major role in encouraging exclusive breastfeeding.
\end{abstract}

Key Words: Breastfeeding, Societal factors, Perceptions, New mum

\section{INTRODUCTION}

Breastfeeding (BF) is an optimal approach for infant feeding capable of stimulating short and long term benefits for both babies and their mothers. It ensures healthy infant growth and development at an early stage in life, supplying the infants' bodies with antibodies that protect them from many common childhood illnesses, as well as ensuring healthier body weight, and lower body mass index. ${ }^{[1,2]}$ Ideal breast- feeding practices, as recommended by WHO, involve the early initiation of BF immediately after infant's delivery, with exclusive breastfeeding for the first six months of life and then the continuation of breastfeeding complemented with solid food for up to two years of age. ${ }^{[3]}$

Exclusive BF means that the infant is fed only with breast milk and does not take any other food, except medications and vitamins supplements if needed. These recommenda-

*Correspondence: Fahima Al Harthy; Email: kemoharthy@gmail.com; Address: Royal Hospital, Ministry of Health (MOH), Oman. 
tions are supported strongly by numerous medical and professional organizations, such as the American Academy of Paediatrics (AAP), the American Academy of Family Physicians (AAFP), the American College of Obstetricians and Gynaecologists (ACOG), the Canadian Paediatric Society (CPS), and the World Health Organization (WHO). ${ }^{[1]}$

Evidence shows that breastfed children have at least a six times greater chance of survival in the early months compared to non-breastfed children. BF considerably reduces deaths from diarrhoea and acute respiratory infection, as well as from other infectious diseases. ${ }^{[4]}$ Furthermore, mothers who do not breastfeed have an increased risk of developing premenopausal breast cancer, ovarian cancer, myocardial infarctions, retained gestational weight gain, type 2 diabetes, and different metabolic syndromes. ${ }^{[5,6]}$

Globally 3 out of 5 babies are not breastfed in the first hour of life, only $41 \%$ of all infants under 6 months of age are exclusively breastfed and not more than $45 \%$ of children remain breastfed at 2 years of age. ${ }^{[4]}$ In the Middle East, $\mathrm{BF}$ rates are even lower and do not attain the target level set by the WHO, with rates declining from $30 \%$ in 1990 to $26 \%$ in $2006 .{ }^{[7]}$ A family health survey conducted in UAE indicated that only $34 \%$ of infants were exclusively breastfed for up to 4 months of age. ${ }^{[8]}$ Oman, in particular, has BF indicators that are suboptimal despite the implementation of BFHI: ${ }^{[7]}$ the rate of exclusive BF at birth was $97.5 \%$ in 2005 but decreased to $94.9 \%$ in 2012 ; only $31.3 \%$ of infants were exclusively breastfed for six months in 2005 , and $23.2 \%$ in 2017. Overall, the total number of children who were ever breastfed until 24 months were $96.1 \%$. ${ }^{9]}$

According to a study conducted in UAE, ${ }^{[10]}$ understanding mothers' BF perceptions and experiences are recognized as vital tool to give effective support that would encourage the extension of the exclusive BF period. Hence, this study aims to explore the perception, beliefs and societal factors influencing exclusive BF practices.

\section{RESEARCH DESIGN AND METHODOLOGY}

This qualitative exploratory study was conducted at the largest tertiary health care hospital in Oman, from March until November 2020. For the study, ten primiparous mothers were recruited with specified inclusion criteria (newly delivered mothers after 24 hours of delivery and of Omani nationality). Purposive sampling was applied during the recruitment and face to face interviews were applied for data collection. This approach was designed to allow the mothers to freely express their perceptions, beliefs and societal factors influencing their BF practices.

The study was conducted within the post-natal wards in the Published by Sciedu Press
Royal Hospital. Each participant was evaluated for any risk of bleeding, pain and discomfort before their interview to ensure maximum engagement and maintain a safe environment. Mothers that did not satisfy the criteria were excluded from the study.

Two researchers were assigned for data collection: one was the main interviewer and the other was the data recorder. The two researchers were trained by a lead researcher who is an expert in qualitative research and possesses extensive experience in qualitative research, face to face data interviews and data collection. The interview protocol was structured based on research objectives and reviewed by the research team to ensure appropriate guidance during the interviews with a standardized flow of questions and appropriate prompts. All interviews were conducted in Arabic as all participants were Arabic native speakers. The interview protocol was prepared in English, translated to Arabic and then reverse translated by a native Arabic speaker. The protocol included demographic data such as age, place of residence, and employment status. Additional information was collected about mothers' knowledge on exclusive BF, their sources of information, influencing factors and their intention towards their breastfeeding practices. Each face to face interview lasted for 40-60 minutes.

Methodological consideration; all interviews were audiorecorded and transcribed verbatim. Moreover, to ensure maximum comfort of the mothers during the interviews, all participants were interviewed anonymously and individually in a separate room and were allowed to feed their babies before the interview to avoid interruption.

\subsection{Data analysis}

Thematic analysis was applied for data analysis. In the first analysis round, all researchers independently analysed the transcripts and all shared their analysis with the rest of the team. The second round of analysis was done after the agreement of the themes, sub-themes and categories. In the third round of analysis, the lead researcher set the themes definitions, sub-themes and categories. A total of six themes were developed:

(1) The effects of lack of knowledge on the mother perception towards exclusive BF

(2) Family members influencing the decision-making process regarding $\mathrm{BF}$

(3) Impact of social life on BF practices

(4) The effect of mother's beliefs on exclusive BF

(5) Factor that affect BF practice in early days

(6) Mother's concern regarding emotional support for successful exclusive $\mathrm{BF}$ 


\subsection{Ethical considerations}

This study was approved by the ethical committee of Royal hospital SRC \#1/2020. Participants voluntarily signed in formed consent before the interviews and were assured that they have the right to voluntary withdrawal at any point during the study. All interviews were conducted in a private room to ensure the participants' comfort. After the interviews' transcription, all records were subsequently discarded.

\section{RESULTS}

The participants involved were newly delivered mothers with a first child, all of them falling within the 25 and 33 years age bracket, with eight of them having received a university education and only two with basic education. Eight of the in terviewees were housewives and two of them were formally employed.

\subsection{The effects of lack of knowledge on the mother per- ception towards exclusive BF}

Limited knowledge and understanding of exclusive BF, coupled with an unawareness of the National Breastfeeding Policy (ten steps of successful breastfeeding) were elicited in most of the participants. The participants expressed their opinions as follows:

"This is the first child and I don't know about exclusive breastfeeding."

The mothers declared that the majority of information they possessed had been received from social media and the in ternet. Some of them added that to learn more about BF they had to depend on their relatives who have previous experience with breastfeeding.

\subsection{Family members influencing the decision-making process regarding $\mathrm{BF}$}

An influencing member is defined as a person or someone that has an impact on shaping how people act- that is, a person who convinces others to listen to or do as they suggest. Most of the mothers verbalize that they are influenced by family members such as the grandmother and husband. Mothers expressed that family opinions, especially the infant's grandmother, can affect their BF decision because grandmothers generally have experience and they could know more about $\mathrm{BF}$ than them.

"My mother helps me, I think without her help I can't feed. My husband also, helps me when I need it", said one interviewee.

Another participant stated "members within my family play an important role about breastfeeding decision, even on the choice whether to continue the breastfeeding or not".

\subsection{Impact of social life on BF practices}

Social life refers to an individual's interpersonal relationship within their immediate surrounding or the general public. Most of the interviewed mothers articulated significant concerns regarding breastfeeding their babies in public places such as public malls, parks, beaches, cinemas, supermarkets and airports. One of the mothers suggested that there should be private places to breastfeed within public settings "Not preparing a suitable place for breastfeeding at the malls and other public areas is a major obstacle that prevents mothers from breastfeeding".

According to conservative Arabic culture, it is unacceptable to expose the breast and feed in front of others. Visitors, neighbours, and friends have varying influences on these new mothers. One mother said: "In the community, there are two categories, those who support and those who do not. But most of them are encouraging me to breastfeed." Another mother said: "Positive attitude from the community is one of the factors that helps a mother to exclusively breastfeed, but sometimes the community gives negative messages." Some mothers feel the community plays a major role in helping them breastfeed exclusively as they are more experienced. As one participant expressed: "The visitors keep asking me, why not to give formula milk, your milk is not enough."

Furthermore, when guests visit, mothers feel embarrassed to breastfeed in front of them, which may lead to the decision for bottle feeding. One mother said: "In our community, lots of visitors are coming and the mother gets tired and sometimes she is embarrassed, so she gives formula feed."

\subsection{The effect of mother's beliefs on exclusive BF}

Mothers have certain positive beliefs regarding exclusive BF. They believe that breastfeeding increases the bonding between the mother and her baby. These beliefs aid in the decision of selecting BF instead of bottle feeding.

"I believe that breastfeeding is good for the mother's wellbeing, both mentally and physically, and it enhances bonding between the mum and her baby."

\subsection{Factor that affect BF practice in early days}

Mothers have negative perceptions regarding breastfeed their babies during the colostrum phase as they feel that its amount is not adequate for the baby. As one mother mentioned, "I don't feel my milk is enough for my baby as I just delivered yesterday." Participants had serious concerns with regards to the physical discomfort in the early days after delivery, milk adequacy and their knowledge about BF techniques. In terms of physical fatigue, mothers expressed that "breastfeeding is exhausting in the beginning and the baby needs time till they get used to it". In addition, the mothers had a major concern 
about milk adequacy that led them to decide to add artificial milk formula feeds to their babies, which is considered one of the restricting factors for exclusive BF. "If I found that my baby does not get enough milk I will ask for formula feeds, I am afraid that my baby blood sugar gets low."

It has also been observed that mothers face uncertainties which lead to difficulties to sustain BF as it is a new experience "In the first few days, it was difficult to breastfeed my baby because I don't know much about breastfeeding”.

\subsection{Mother's concern regarding emotional support for successful exclusive BF}

Emotional status is referring to a person's feeling of pleasure or dejection. The emotional stability of the mother plays a major role in deciding whether to breastfeed exclusively or not. As one participant mentioned: "psychological stability is important to decide for breastfeeding or not." Most of the mothers expressed their feelings about the importance of their psychological comfort to succeed in exclusive BF. As one mother stated: "The mother needs to be stable in terms of feeling and reaction." Another mother expressed: "I need the healthy food and positive feelings to be comfortable."

Comfortable and happiness is one of the positive attitudes that lead the mother to be able to feed. As one mother mentioned: "I feel happy when I see my baby is getting breastfed."

On the other hand, feelings of embarrassment, fear, tiredness, sickness or sadness can be an obstacle for BF. As one mother mentioned: "mental status of the mother caused by a negative situation or sickness-for example, corona- will affect the breastfeeding." Another mother expressed "If the mother is not comfortable, she will not be interested to breastfeed". To add more, the loss of beloved ones in the family can affect the sentient status. As one mother mentions "if the mother is stressed such as being sick or lost a family member, these are a barrier to breastfeeding".

Furthermore, there are some factors leading to negative attitudes such as when a mother doesn't know how to position her baby for BF but after addressing the concerns most of the psychological factors will be resolved. As one mother mentioned "My baby is in NICU and she did not get breastfeeding directly, immediately she sleeps when I try to feed her, I think this is a problem. Now after learning from breastfeeding nurse I felt things are getting better."

\section{DisCUSSION}

This study revealed that most mothers lack a good understanding of the definition of exclusive BF and the ten steps of successful BF. In addition, they have perceptions of sig- nificant barriers for exclusive BF such as feeding in public places, family influences, and the difficulties associated with BF in the early days. The novelty of this study is the strong overarchingly positive belief in the importance and benefits of breast milk regardless of all negative influences from the community. These findings build on, validate, and contribute to the results of other studies on exclusive BF.

\subsection{Knowledge of exclusive breastfeeding}

This study revealed that mothers are significantly lacking knowledge of exclusive BF. This means that they either do not know what is meant by exclusive $\mathrm{BF}$, duration, or the ten steps of exclusive BF that were defined by WHO. Although all interviewed mothers were highly educated, this lack of knowledge was trending during interviews. This may reflect the lack of support at prenatal clinics and qualified lactation nurses. The lack of support from health system in regards to knowledge of exclusive BF was supported by Mahdi et al. ${ }^{[11]}$ as in this study population usually mothers during pregnancy follow up at Primary health care settings where there is no lactation consultants available.

Nonetheless, mothers undertaking prenatal visits did not benefit from adequate preparation in terms of BF sessions as it was evident from interviews. This is important, in accordance with Piro, ${ }^{[12]}$ given that BF education during pregnancy increase $\mathrm{BF}$ initiation after birth. As the majority of mothers reported the use of social media and the internet for information seeking during pregnancy, then this issue of lacking knowledge can be improved through the maximization of social media use in prenatal visits. These findings are supported by Nasrabadi et al. ${ }^{[13]}$ that social media and internet is a trusted source of information for pregnant women regarding breastfeeding. Therefore, more training for nurses at prenatal clinics are highly important to increase the support provided for mothers in regards to breastfeeding. Moreover, lactation nurses are needed within PHC settings where ANC occurs.

\subsection{The most influencing member in the family}

The findings of this study showed that the husband and grandmother (grandmother of the infant) are the most influencing members within the family for BF decisions. However, the influence could be due to the trust of mothers toward their mothers and husbands. Moreover, the new mother usually seeks advice from those who are considered experts in her point of view. Therefore, grandmothers are being viewed as experts in BF within the family, although they are not involved during prenatal follow up. This is with alignment of Radwan ${ }^{[10]}$ and Nasrabadi et al., ${ }^{[13]}$ which indicated that grandmothers and husbands can affect exclusive BF deci- 
sions either positively or negatively. Therefore, there is a need to target and involve relevant family members in prenatal counselling sessions.

\subsection{Social life impact on breastfeeding}

This study recognizes that societal factors and local culture play a major role in exclusive BF decisions. This includes the cultural values and beliefs after delivery and social interaction as well as the community structure and views towards $\mathrm{BF}$, this was supported as well by Nasrabadi et al. ${ }^{[13]}$ that cultural values have either positive or negative effect on BF For example, during the first month after delivery, visitors come to visit the mother and they are usually family members or neighbours. Interviews revealed that the majority of mothers have huge concerns in undertaking BF in front of visitors or public places, commenting that it is not always acceptable or embarrassing. Moreover, social factors, including friends have a major effect on BF. This means that each community have specific values toward $\mathrm{BF}$ in particular and child-raising in general. This social effect on BF was supported by Santacruz-Salas et al. ${ }^{[2]}$ as mother do not achieve optimal expectation of BF due to social environment . Mothers have concerns as they seek advice from experts in their opinions and adopt the same beliefs as the community. Some of the negative beliefs such as inadequate milk production or risk of jaundice lead mothers to decide on adding supplementary feed just to feel satisfied that the baby is getting enough milk. Therefore, it is considered important to engage communities and ensure the availability of BF corners in public settings.

\subsection{Mothers' beliefs and trust in exclusive breastfeeding}

This study showed that regardless of all negative messaging emanating from the community, all interviewed mothers have strong trust in breastfeeding in terms of infant immunity, health, positive mother-baby bonding and emotional stability, growth and disease prevention in the future. Moreover, they have a very strong belief that since breast milk is created by GOD, it is certainly better than any other milk. These beliefs support the decision of choosing BF instead of bottle feeding. These findings are in accordance with Wanjohi ${ }^{[14]}$ that positive mothers' beliefs aid in the decision of choosing BF.

\subsection{Early days after delivery}

This study showed that in the early days after delivery, mothers have negative emotions toward initiation of BF due to several factors as stated by mothers.

These factors include perceptions regarding inadequacy in the amount of milk, ${ }^{[13,15,16]}$ physical discomfort or fatigue, and poor knowledge about BF positioning and attachment techniques. In terms of the amount of milk, mothers stated that they believed their milk is not enough; therefore they tend to introduce formula milk. This could be done by training health staff to better counsel mothers on messaging around early initiation, correct positioning and attachment.

In regards to physical comfort and fatigue, the mothers expressed that this is also influenced by pain from either episiotomy or caesarean and exhaustion after delivery. In addition, lack of knowledge regarding BF techniques is due to the inexperience of mothers in BF as they are new mothers. This could be related to the inadequacy of training and educational session in prenatal visits as supported by Nasrabadi et al. ${ }^{[13]}$ and Neji. ${ }^{[17]}$

\subsection{Mother's concern of emotional comfort for success- ful exclusive BF}

This study indicated that the emotional status of the mother is one of the factors which influence milk production and the mother's decision whether to breastfeed exclusively or not. This implies that being emotionally stable, in terms of being happy and comfortable, will positively influence the mother's ability to exclusively BF. On the other hand, being emotionally upset, stressed, and fatigued will negatively affect the exclusive BF through the effects of prolactin and oxytocin hormones, this was supported as well by Emilda ${ }^{[15]}$ and Mensah. ${ }^{[16]}$ Therefore, women will have inadequate milk production and this is the reason for early supplementation. This finding supports the previous literature that emotional status affects the mother ability to exclusive BF. ${ }^{[13]}$ However, these negative emotions can be tackled by psychological support to mothers.

\section{Conclusions}

This study adds to the existing literature on beliefs and perceptions of the Arabic culture on exclusive BF. It revealed that newly delivered mothers lack adequate knowledge on the meaning of exclusive BF, and the national Breastfeeding Policy (10 steps of breastfeeding policy). Moreover, grandmothers and husbands were found to be the most influencing members to decide on exclusive BF. The mothers were having major concerns of milk inadequacy in the first few days and expressed that emotional support plays a major role in BF.

However, this study has many limitations. First of all, it is a qualitative study that cannot be generalized to the whole population of Oman. Furthermore, this study was conducted in one tertiary centre in the capital city. This brings attention to the need of exploring the general public perception of exclusive BF which include primi and multipara mothers, grandmothers, and the community.

ISSN 2324-7940 E-ISSN 2324-7959 


\section{ACKNOWLEDGEMENTS}

The authors would like to acknowledge the Royal Hospital administration for the support in conducting the study. Bushra Al Maskri, Dr Huwaishal Al Lamki, Manal Al Ajmi for English review.

\section{CONFLICTS OF INTEREST Disclosure}

The authors declare they have no conflicts of interest.

\section{REFERENCES}

[1] Couto GR, Dias V, Oliveira I de J. Benefits of exclusive breastfeeding: An integrative review. Nurs Pract Today. 2020; 7(4): 245-54. https://doi.org/10.18502/npt.v7i4.4034

[2] Santacruz-Salas E, Aranda-Reneo I, Segura-Fragoso A, et al. Mothers' expectations and factors influencing exclusive breastfeeding during the first 6 months. Int J Environ Res Public Health. 2020; 17(1): 3-5. PMid: 31861896. https://doi.org/10.3390/ijer ph17010077

[3] Meek JY, Feldman-Winter L, Noble L. Optimal Duration of Breastfeeding. Pediatrics. 2020 Nov; 146(5): e2020021063. PMid: 33097656. https ://doi.org/10.1542/peds . 2020-021063

[4] WHO. Infant and young child feeding. 2021. 46-7 p.

[5] Sloand E, Budhathoki C, Junn J, et al. Breastfeeding among Latino Families in an Urban Pediatric Office Setting. Nurs Res Pract. 2016; 2016: 1-6. PMid: 27980866. https ://doi.org/10.1155/2016 /9278401

[6] Victora CG, Bahl R, Barros AJD, et al. Breastfeeding in the 21st century: Epidemiology, mechanisms, and lifelong effect. Lancet. 2016; 387(10017): 475-90. https://doi.org/10.1016/S014 0-6736 (15) 01024-7

[7] Al-Nuaimi N, Katende G, Arulppan J. Breastfeeding trends and determinants. Sultan Qaboos Univeristy Med J. 2017; 17: 15561. PMid: 28690886. https://doi.org/10.18295/squmj . 201 6.17 .02 .004

[8] Taha Z, Garemo M, Nanda J. Patterns of breastfeeding practices among infants and young children in Abu Dhabi, United Arab Emirates. Int Breastfeed J. 2018; 13(1): 1-10. PMid: 30479650. https : //doi .org/10.1186/s13006-018-0192-7

[9] National O, Survey N. Oman Nutrition Survey 2017. 2018; 2017.

[10] Radwan H, Sapsford R. Maternal Perceptions and Views about Breastfeeding Practices among Emirati Mothers. Food Nutr Bull. 2016;
37(1): 73-84. PMid: 26793991. https://doi.org/10.1177/03 79572115624289

[11] Mahdi AY, Nzioki JM, Kubende P. Prevalence, Level of Awareness and Socio-Demographic Predictors of Exclusive Breastfeeding among Women of Reproductive Age in Waberi District, Mogadishu. Afr J Health Sci. 2020; 33(5): 110-7.

[12] Piro SS, Ahmed HM. Impacts of antenatal nursing interventions on mothers' breastfeeding self-efficacy: An experimental study. BMC Pregnancy Childbirth. 2020; 20(1): 1-12. PMid: 31906881 https://doi.org/10.1186/s12884-019-2701-0

[13] Nasrabadi M, Vahedian-Shahroodi M, Esmaily H, et al. Factors affecting Exclusive breastfeeding in the first six months of birth: An Exploratory-Descriptive Study. J Midwifery Reprod Heal. 2019; 7(3): 1749-64.

[14] Wanjohi M, Griffiths P, Wekesah F, et al. Sociocultural factors influencing breastfeeding practices in two slums in Nairobi, Kenya. Int Breastfeed J [Internet]. 2017; 12(1): 1-9. PMid: 28096888. https : //doi .org/10.1186/s13006-016-0092-7

[15] Emilda E, Juliastuti J. The effectiveness of oxytocin and marmet massage on increased prolactin hormone for smooth breastfeeding in postpartum mothers in langsa city health office, Indonesia. Open Access Maced J Med Sci. 2020; 8(E): 578-81. https : //doi.org/10.3889/oamjms. 2020.4739

[16] Mensah KA, Acheampong E, Anokye FO, et al. Factors influencing the practice of exclusive breastfeeding among nursing mothers in a peri-urban district of Ghana. BMC Res Notes. 2017; 10(1): 1-7. PMid: 28882162. https://doi.org/10.1186/s13104-017-2 774-7

[17] Neji ON, Chidiebere CC. Exclusive Breastfeeding perception and practice among nursing mothers attending infant welfare clinic in a secondary health facility in Southern Nigeria. African J Heal Nurs Midwifery. 2019; 2(1): 22-34. 